\title{
Ectopic Varices at Hepaticojejunostomy Site Presenting as Obscure Gastrointestinal Bleed with Portal Biliopathy
}

\author{
Arun Devjibhai Solanki ${ }^{1}$, Shubha Immaneni ${ }^{2}$, Aravind A. ${ }^{3}$, Caroline Selvi ${ }^{4}$, \\ Premkumar Karunakaran ${ }^{5}$, Chezhian Annasamy ${ }^{6}$ \\ 1, 2, 3, 4, 5, 6 Institute of Medical Gastroenterology, Madras Medical College, Chennai, Tamilnadu, India.
}

\section{ABSTRACT}

\section{RATIONALE}

Ectopic varix at the hepaticojejunostomy site is a rare finding. So symptomatic jejunal varices at this site are difficult to manage.

\section{PATIENT CONCERN}

A 37-year-old female presented with bleeding per rectum for 5 months with a history of hepaticojejunostomy done 6 years back for management of postcholecystectomy bile leak.

\section{DIAGNOSIS}

The endoscopic investigation could not identify the source of bleeding. When conservative management could not control her bleeding, she underwent DSA which showed haemorrhage from ectopic varices at the hepaticojejunostomy site.

\section{INTERVENTION}

The patient underwent embolization of ectopic varices to arrest bleeding

\section{OUTCOME}

After embolization, patient bleeding per rectum stopped and improvement in haemoglobin was noted within 3 days of the procedure.

\section{ABBREVIATIONS}

DSA: Digital subtraction angiography, SMA: Superior mesenteric artery, SMV: Superior mesenteric vein, HJ: Hepaticojejunostomy, AVSC: Adult variable stiffness colonoscope, SBE-Single balloon enteroscopy, DBE: Double balloon enteroscopy

\section{KEY WORDS}

Hepaticojejunostomy, Portal Biliopathy, Ectopic Varices, Variceal Embolization.
Corresponding Author: Dr. Arun Devjibhai Solanki, MMC PG Mens Hostel, Esplanade, Park Town, Chennai Central, Chennai, Tamilnadu, India. E-mail:aurosolanki@gmail.com

DOI: $10.14260 / \mathrm{jemds} / 2022 / 84$

How to Cite This Article:

Solanki AD, Immaneni S, Aravind A, et al. Ectopic varices at hepaticojejunostomy site presenting as obscure gastrointestinal bleed with portal biliopathy. J Evolution Med Dent Sci 2022;11(03):431-435, DOI: 10.14260/jemds/2022/84

Submission 10-01-2022,

Peer Review 04-02-2022,

Acceptance 10-02-2022,

Published 25-02-2022.

Copyright (C) 2022 Arun Devjibhai Solanki et al. This is an open access article distributed under Creative Commons Attribution License [Attribution 4.0 International (CC BY 4.0)] 


\section{INTRODUCTION}

The small intestine is not the common site for the development of varices. Diagnosis of small intestinal varices is difficult as they are not accessible by a normal upper GI endoscope. Moreover, the majority of intestinal varices are often accompanied by extrahepatic portal vein obstruction, which is found to be associated with intra-abdominal adhesions secondary to trauma or some previous surgery. Therefore, it becomes very difficult to perform any operative obliteration of this varix and also shunt surgery is difficult as these areas are highly vascular. We report a case of ectopic jejunal varices at the hepaticojejunostomy site which is not associated with extrahepatic portal vein obstruction and presented as obscure GI bleed with portal biliopathy. These varices were managed by transcatheter embolization to occlude the collaterals from the jejunal vein supplying the hepaticojejunostomy site.

\section{Case Report}

A 37-year-old female presented with complaints of bleeding per rectum for the last 5 months not associated with hematemesis. Six years before this presentation, the patient had undergone laparoscopic cholecystectomy in January 2015. Post-procedure the patient developed abdominal pain not relieved with medications and was found to have developed postcholecystectomy bile leak for which the patient initially was managed by ERCP with CBD stenting but due to persistent bile leak, the patient underwent hepaticojejunostomy (HJ) on $24^{\text {th }}$ February 2015. The patient was doing well for initial 11 months posthepaticojejunostomy, after which she developed jaundice and abdominal pain and on evaluation, the patient was found to have developed an anastomotic benign stricture at the $\mathrm{HJ}$ site for which the patient underwent percutaneous biliary drainage along with balloon dilatation of stricture at $\mathrm{HJ}$ site on $16^{\text {th }}$ February 2016.

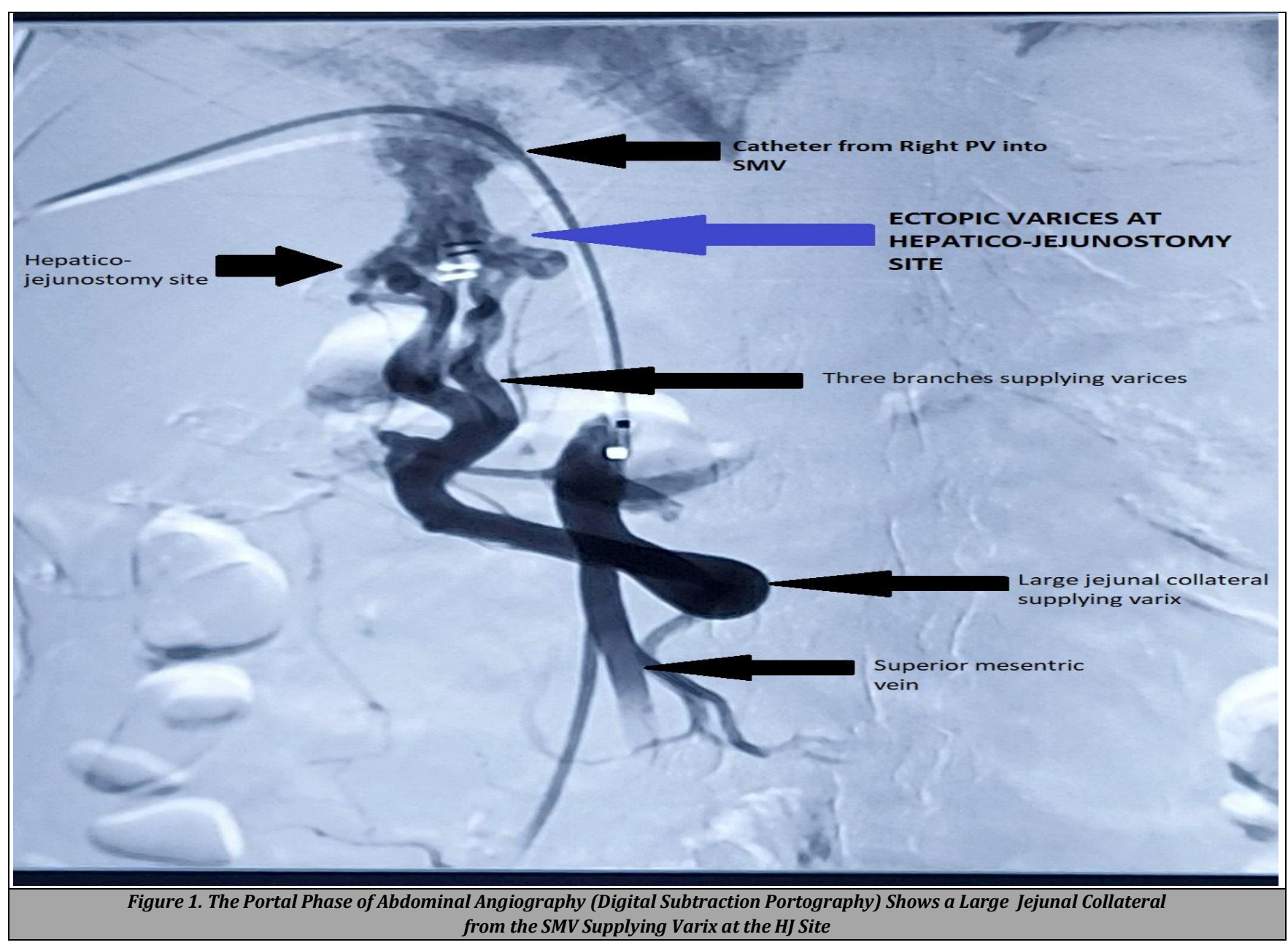

The patient was finally discharged and was on regular follow up. The patient was doing well until 5 months back, when she presented with complaints of repeated episodes of hematochezia requiring hospitalization for blood transfusion along with upper and lower gastrointestinal endoscopy. Upper GI endoscopy only revealed 3 diminutive polyps, antrum inflamed with no oesophagal or gastric varices and no ulcers. Colonoscopy showed features suggestive of proctosigmoiditis and was treated with mesalamine, prednisolone but the patient continued to have bleeding PR and so the patient was advised to stop mesalamine and prednisolone. USG abdomen was suggestive of chronic liver disease with features of portal hypertension. The most likely cause of this cirrhosis was the stricture at the anastomotic site of hepaticojejunostomy. Multidetector CT Abdominal angiogram revealed features suggestive of Hepatic AV malformation with collaterals from left hepatic artery supplying right lobe of the liver, collaterals noted at the level of hilum draining into SMV and thrombosed left portal vein. Technetium-99m-labelled red cell scintigraphy also did not 
show any abnormality. The patient further underwent digital subtraction angiography (DSA) that revealed on the delayed phase of SMA run showed predominant flow from SMV into large jejunal collateral which was contributing to the $\mathrm{HJ}$ site varices and few collaterals were seen directly into liver parenchyma from the $\mathrm{HJ}$ site and the $\mathrm{AV}$ malformation seen on CT Abdominal angiogram were tortuous collaterals of left hepatic artery as shown in DSA, Main portal vein, Right portal vein were normal on DSA but left portal vein was thrombosed as it was not opacified on transsplenic portogram. The patient was given symptomatic treatment with IV antibiotics, blood transfusion, octreotide but still, the patient continued to have a drop of haemoglobin and developed portal biliopathy due to compression of the $\mathrm{HJ}$ site by the varices and so was planned embolization of varices at the $\mathrm{HJ}$ site. Under SAP, a guidewire and catheter were passed into SMV through the right portal vein and entered the large jejunal branch supplying the varices at the $\mathrm{HJ}$ site and a mixture of glue and lipiodol was injected into the three branches supplying varix. Postprocedure, there was no further bleeding and patient bilirubin decreased drastically from direct bilirubin of 19 $\mathrm{mg} / \mathrm{dl}$ to $2.1 \mathrm{mg} / \mathrm{dl}$ within 3 days along with significant improvement in haemoglobin from $5.2 \mathrm{gm} / \mathrm{dl}$ to $8.7 \mathrm{gm} / \mathrm{dl}$. One day after the procedure, the patient underwent screening USG abdomen with Doppler showing significant reduction in the collaterals at $\mathrm{HJ}$ site with reduced flow, ectopic varices at $\mathrm{HJ}$ site were not visualised and minimal flow in the jejunal collateral vein. The patient was clinically stable with improving haemoglobin, normalising bilirubin and with no complaints of bleeding per rectum post-procedure.

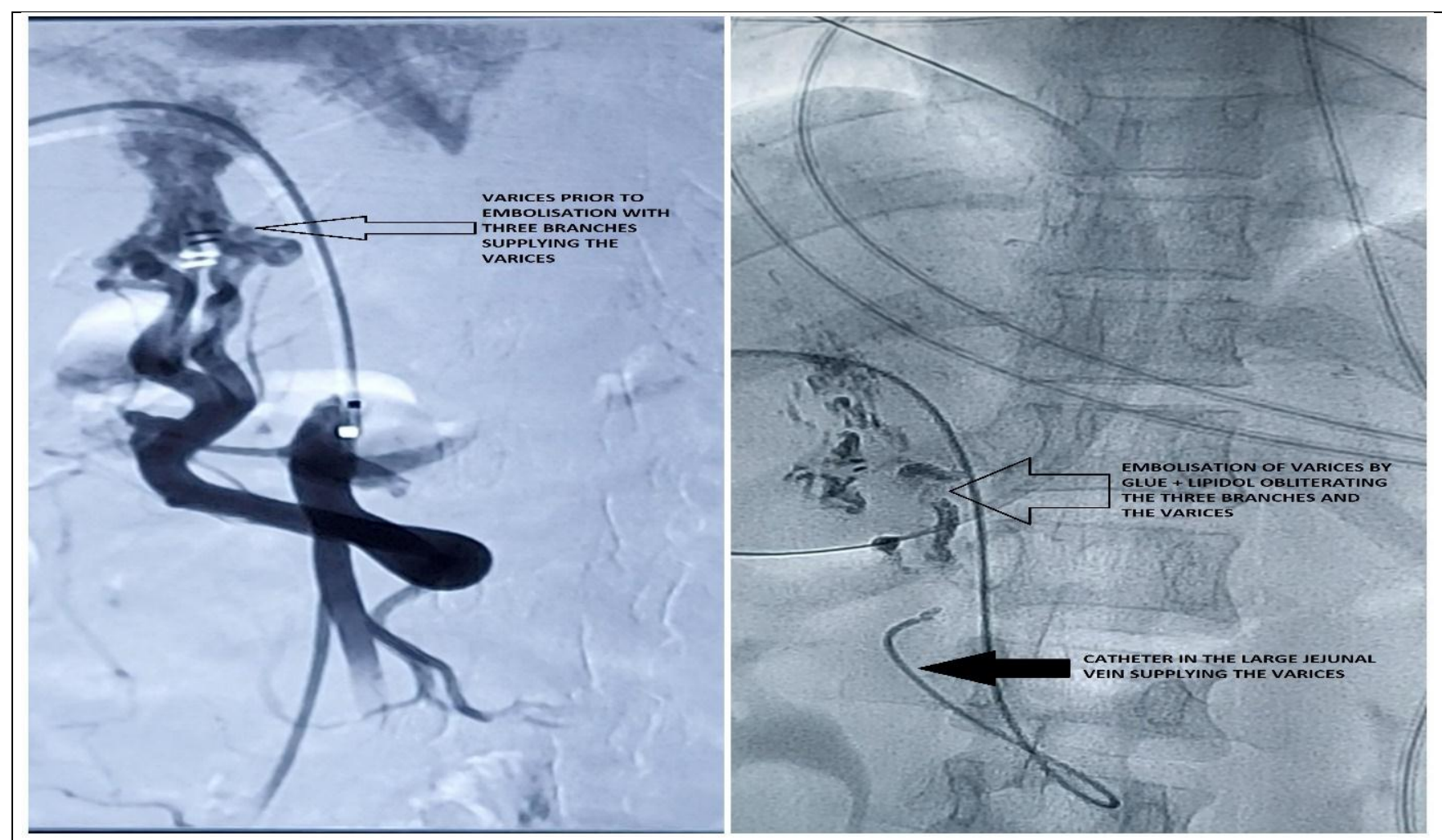

Figure 2. Pre- \& Post Embolization of Varices Showing Complete Obliteration of Varices by the Mixture of Glue + Lipiodol Inserted into the Varices

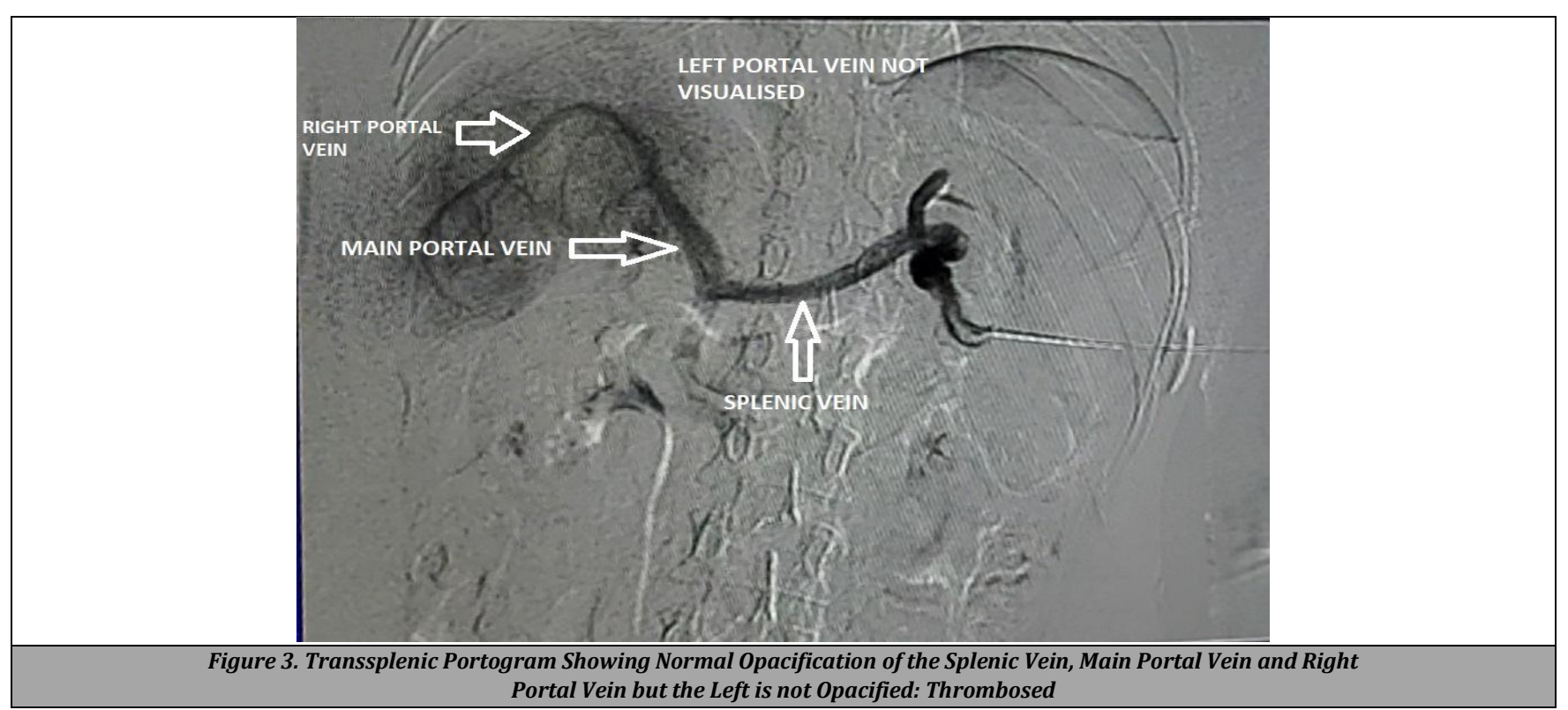




\section{DISCUSSION}

Ectopic varices are uncommon and it accounts for less than 5 $\%$ of cases of variceal bleeding. ${ }^{1,2}$ Variceal bleed from the HJ site almost occurs secondary to portal vein stenosis or thrombosis which leads to the development of portal hypertension. Other reasons implicated are due to repeated inflammation at the site of anastomosis like recurrent retrograde cholangitis due to narrowing or stricture, bile leak at the anastomotic site or some tumour invading or compressing. The exact reason why varices develop at the site is still not clear. . $^{3,4}$

$1^{\text {st }}$ episode of bleeding may take months to years from the initial surgery, as seen in our case the patient developed bleeding after a span of 6 yrs from $\mathrm{HJ}$.

In our case, although the main portal vein was patent the left portal vein was thrombosed, there was development of the collateral at the $\mathrm{HJ}$ site, in which multiple thin collaterals from the $\mathrm{HJ}$ site entered in the left lobe of the liver which communicated with the jejunal branch of SMV of the jejunal afferent loop.

Management of ectopic varices at the $\mathrm{HJ}$ site will involve two types of treatment; either obliteration of varix responsible for bleeding or shunt surgery. The aim of obliteration of the varix is to stop blood flow to the varix, but there will be a possibility of rebleeding from newly developed varices that remain because the procedure does not correct portal hypertension. Whereas shunt surgery aims for portal system decompression to prevent bleeding and it also prevents the formation of new varices. However, rebleeding can still occur from the same varix, if the shunt flow is not adequate to decompress for the portal system. ${ }^{5}$

Merits of embolization of varices: Reduced surgical insult, embolization point can be selected by portography as it allows to stop the inflow of blood to the varices at a point near the varices and can help to prevent possible relapse of varix, to predict hemodynamic changes which accompany the embolization and also which may provide information about possible relapse and maintain haemostasis. Therefore, embolization of intestinal varices using an interventional radiology technique is a safe and effective treatment for intestinal bleeding. 6

Endoscopy is the ideal approach for rapid diagnosis and immediate management for bleeding from the afferent jejunal loop of hepaticojejunostomy. However, due to altered anatomy due to surgery, it would not be possible to reach the jejunal afferent loop with conventional gastroduodenoscopy. Therefore, a longer endoscope is needed to access the loop of Roux-en-Y. Compared with conventional gastroduodenoscopy, a single-balloon endoscope (SBE) $7,8,9$ and double-balloon endoscope (DBE) have more length and also have balloon overtube to insert more deeply. SBE and DBE seem to be equally useful for diagnostic and therapeutic procedures. ${ }^{10,11}$ However, these specialized devices are not easily and always available at all institutions.

The adult variable stiffness colonoscope (AVSC) can be used in place of SBE or DBE if not available to access the lumen of the gastrointestinal tract beyond the duodenum as well as to enter the Roux-en-Y jejunal loop for diagnostic as well as therapeutic purposes.

Literature that describes the use of AVSC for upper GI investigation and treatment, particularly for Roux-en-Y anatomy, are not found easily. Chahal et al. have reported in endoscopic retrograde cholangiography for post-orthotopic liver transplant population with Roux-en-Y biliary anastomosis, the usefulness of AVSC. ${ }^{12}$ Azeem et al. compared paediatric colonoscope, SBE with AVSC and concluded that AVSC has a less procedural success rate as compared to SBE. ${ }^{13}$ AVSC can be an alternative device for SBE and DBE but because it has a diameter which is larger than balloon enteroscopy and it is much stiffer than the conventional gastroduodenoscopy, it may be stressful for the patient's throat. Neumann et al. first reported the treatment using endoscopy in a patient with pancreatic cancer whose portal vein was obstructed 1 year after the operation. DBE was used to gain access to the afferent loop and haemostasis was achieved using Argon plasma. ${ }^{14}$ Prachayakul et al. used SBE and varix was obliterated using an injection of histoacryl, and although successfully done, thrombosis at the branch of the superior mesenteric vein occurred. ${ }^{15}$ Curcio et al. used SBE and endoclips for haemostat, but after the clip placement, it led to the rupture of varix and cyanoacrylate injection was given to control bleeding. ${ }^{16}$ Therefore, out of reach bleeding can be managed even without the use of advanced endoscopes.

\section{CONCLUSIONS}

Ectopic varices at the hepaticojejunostomy site are not accessible through conventional gastroduodenoscopy, they require special endoscopes like SBE or DBE or by using AVSC. Embolization of these varices by interventional radiology is a safe and effective method to deal with these ectopic varices and shunt surgery should be reserved for those patients who fail to respond to other therapies.

\section{REFERENCES}

[1] Salam AA, Goldman M, Smith D, et al. Gastric, intestinal, and gallbladder varices: hemodynamic and therapeutic considerations. South Med J 1979;72(4):402-8.

[2] Wilson SE, Stone RT, Christie JP, et al. Massive lower gastrointestinal bleeding from intestinal varices. Arch Surg 1979;114(10):1158-61.

[3] Moncure AC, Waltman AC, Vandersalm TJ, et al. Gastrointestinal hemorrhage from adhesion-related mesenteric varices. Ann Surg 1976;183(1):24-9.

[4] Furugaki K, Yoshida J, Hashizume M, et al. The development of extrahepatic portal obstruction after undergoing multiple operations for a congenital dilatation of the bile duct: report of a case. Surg Today 1998;28(3):355-8.

[5] Katoh H, Shimozawa E, Kojima T, et al. Modified splenorenal shunt with splenopancreatic disconnection. Surgery 1976;106(5):920-4.

[6] Sato T, Asanuma Y, Ishida H, et al. A case of extrahepatic portosystemic shunt without portal hypertension treated by laparoscopically assisted embolization. Surgery 1999;126(5):984-6.

[7] Saleem A, Baron TH, Gostout CJ, et al. Endoscopic retrograde cholangiopancreatography using a single- 
balloon enteroscope in patients with altered Roux-en-Y anatomy. Endoscopy 2010;42(8):656-60.

[8] Wang AY, Sauer BG, Behm BW, et al. Single-balloon enteroscopy effectively enables diagnostic and therapeutic retrograde cholangiography in patients with surgically altered anatomy. Gastrointest Endosc 2010;71(3):641-9.

[9] Hartmann D, Eickhoff A, Tamm R, et al. Balloon assisted enteroscopy using a single-balloon technique. Endoscopy 2007;39(Suppl 1):E276.

[10] Domagk D, Mensink P, Aktas H, et al. Single- vs. doubleballoon enteroscopy in small-bowel diagnostics: a randomized multicenter trial. Endoscopy 2011;43(6):472-6.

[11] Moreels TG, Pelckmans PA. Comparison between doubleballoon and single-balloon enteroscopy in therapeutic ERC after Roux-en-Yentero-entero anastomosis. World J Gastrointest Endosc 2010;2(9):314-7.

[12] Chahal P, Baron TH, Poterucha JJ, et al. Endoscopic retrograde cholangiography in post-orthotopic liver transplant population with Roux-en-Y biliary reconstruction. Liver Transpl 2007;13(8):1168-73.
[13] Azeem N, Tabibian JH, Baron TH, et al. Use of a singleballoon enteroscope compared with variable-stiffness colonoscopes for endoscopic retrograde cholangiography in liver transplant patients with Rouxen-Y biliary anastomosis. Gastrointest Endosc 2013;77(4): 568-77.

[14] Neumann H, Monkemuller K, Malfertheiner P. Obscure overt GI bleeding secondary to angiodysplasias at the hepaticojejunostomy diagnosed and successfully treated with double balloon enteroscopy. Gastrointest Endosc 2008;67(3):563-5.

[15] Prachayakul V, Aswakul P, Kachintorm U. Bleeding hepaticojejunostomy anastomotic varices successfully treated with histoacryl injection, using single-balloon enteroscopy. Endoscopy 2011;43(Suppl 2):E153.

[16] Curcio G, Sciveres M, Mocciaro F, et al. Out-of-reach obscure bleeding: single-balloon enteroscopy to diagnose and treat varices in hepaticojejunostomy after pediatric liver transplant. Pediatr Transplant 2012;16(3):E78-80. 